\title{
Estimation of Propeller Open-Water Characteristics Based on Quasi-Continuous Method
}

\author{
by Naoto Nakamura*, Member
}

\begin{abstract}
Summary
A numerical method to estimate propeller open-water characteristics based on Quasi-Continuous Method (QCM) is presented. QCM was originally developed by Lan for solving planar thin wing problems. QCM has both advantages of continuous loading method and discrete loading method; loading distribution is assumed to be continuous in chordwise direction and stepwise constant in spanwise direction. Simplicity and flexibility of discrete loading method are also retained.

In the present paper, explanations are made first on the essential features of $\mathrm{QCM}$ for planar wings and then its application to marine propellers is described in detail. Some numerical results on the propeller open-water characteristics for conventional and skewed propellers showed good agreement with those of experiments.
\end{abstract}

\section{Introduction}

In the design of propellers with various blade geometries such as highly skewed propellers, design charts based on methodical series tests are to be complemented by theoretical calculations for accurate estimation of propeller open-water characteristics.

Up to now, a number of methods based on propeller lifting surface theory to estimate openwater characteristics have been published. They can be classified into two groups. One group is based on the continuous loading method such as Mode Function Method (MFM) ${ }^{1-8)}$ and the other the discrete loading method such as Vortex Lattice Method (VLM) $)^{9-11)}$.

In MFM, loading distribution on blades is treated as continuous both in chordwise and spanwise directions and it is beneficial for the estimation of pressure distribution on blades. However, complicated singularity treatment is necessary and the accuracy is affected by the way of singularity treatment ${ }^{6}$. Further, due to the complexity in numerical calculation, its application to unconventional propellers is not easy. On the other hand, VLM has the flexibility and simplicity of calculation, and along with the development of high speed and large storage computer VLM has been widely used in the studies of propeller lifting surface theory. VLM, however, also has some room to be improved;

* Nagasaki Experimental Tank, Mitsubishi Heavy Industries, Ltd.
(1) In the neighbourhood of leading edge, vortex strength predicted by VLM is always lower compared with analytical solutions ${ }^{12}$.

(2) Owing to the discrete and concentrated loading distribution, pressure distribution is not estimated straightforwardly.

(3) Leading edge suction force is not estimated straightforwardly either.

(4) A large number of elements are necessary to get converged solutions.

Taking the above circumstances into consideration, the author initiated to develop a method to estimate propeller open-water characteristics based on Quasi-Continuous Method (QCM) originally developed by Lan for solving planar thin wing problems ${ }^{13)}$. $\mathrm{QCM}$ has both advantages of MFM and VLM, i.e. quasi-continuous loading distribution (continuous in chordwise direction and stepwise constant in spanwise direction) is assumed, therefore it is beneficial for the estimation of pressure distribution on blades. Moreover QCM has the simplicity and flexibility of VLM and its accuracy is comparable to that of NLR method $^{14)}$, a most accurate method among MFM. Owing to continuous loading distribution in chordwise direction, converged solution can be obtained with a small number of control points and leading edge suction force can be calculated directly.

In the present paper, explanations are made first on the essential features of QCM for planar wings and then its application to marine propellers is described in detail. Some numerical results for variety of propellers are compared with those 
of existing methods and experiments.

\section{Characteristics of Quasi- Continuous Method}

Detailed description of QCM was already made by Lan for planar wings ${ }^{13)}$. However, for convenience, a brief explanation of the essential feature of QCM is repeated here. One of the characteristics of $\mathrm{QCM}$ is in the treatment of the continuous loading in chordwise direction. Since the spanwise loading distribution is assumed stepwise constant, we may explain at first the numerical procedure of the chordwise integration by the case of two-dimensional wing. The downwash integral is expressed as follows,

$$
w(\xi)=\frac{1}{2 \pi} \oint_{0}^{1} \frac{\gamma\left(\xi^{\prime}\right)}{\xi-\xi^{\prime}} d \xi^{\prime},
$$

where $\gamma(\xi)$ is vortex strength and $\xi$ is non-dimensional chordwise coordinate. Transforming the $\xi$-coordinate into $\alpha$-coordinate through the relation

$$
\xi=\frac{1}{2}(1-\cos \alpha),
$$

Eq. (1) can be written as

$$
w(\alpha)=-\frac{1}{2 \pi} \oint_{0}^{\pi} \frac{\gamma\left(\alpha^{\prime}\right) \sin \alpha^{\prime}}{\cos \alpha-\cos \alpha^{\prime}} d \alpha^{\prime} .
$$

Denoting further

$$
g(\alpha)=\gamma(\alpha) \sin \alpha
$$

and eliminating the Cauchy singularity in the integrand, Eq. (3) is rewritten as

$$
\begin{aligned}
w(\alpha)= & -\frac{1}{2 \pi} \int_{0}^{\pi} \frac{g\left(\alpha^{\prime}\right)-g(\alpha)}{\cos \alpha-\cos \alpha^{\prime}} d \alpha^{\prime} \\
& -\frac{g(\alpha)}{2 \pi} \oint_{0}^{\pi} \frac{d \alpha^{\prime}}{\cos \alpha-\cos \alpha^{\prime}} \\
= & -\frac{1}{2 \pi} \int_{0}^{\pi} \frac{g\left(\alpha^{\prime}\right)-g(\alpha)}{\cos \alpha-\cos \alpha^{\prime}} d \alpha^{\prime} .
\end{aligned}
$$

Since $g(\alpha)$ does not contain square root singularity at leading edge, the integrand in Eq. (5) is finite everywhere. Thus the integral can be reduced to a finite sum through midpoint trapezoidal rule as follows.

$$
\begin{aligned}
w(\alpha) \cong & -\frac{1}{2 N} \sum_{\nu=1}^{N} \frac{g\left(\alpha_{\nu}\right)-g(\alpha)}{\cos \alpha-\cos \alpha_{\nu}} \\
= & -\frac{1}{2 N} \sum_{\nu=1}^{N} \frac{g\left(\alpha_{\nu}\right)}{\cos \alpha-\cos \alpha_{\nu}} \\
& +\frac{g(\alpha)}{2 N} \sum_{\nu=1}^{N} \frac{1}{\cos \alpha-\cos \alpha_{\nu}},
\end{aligned}
$$

where

$N=$ number of chordwise integration points, $\alpha_{\nu}=\frac{(2 \nu-1)}{2 N} \pi, \quad(\nu=1,2, \cdots \cdots, N)$.
Control points, where flow tangency condition is to be satisfied, are chosen so as to eliminate the second term of Eq. (6) by using a characteristic of Chebychev polynomials as

$$
\sum_{\nu=1}^{N} \frac{1}{\cos \alpha_{j}-\cos \alpha_{\nu}}=0
$$

where

$$
\alpha_{j}=\frac{j}{N} \pi, \quad(j=1,2, \cdots \cdots, N) .
$$

Then the following equation is obtained

$$
\begin{aligned}
w\left(\alpha_{j}\right) & =-\frac{1}{2 N} \sum_{\nu=1}^{N} \frac{g\left(\alpha_{\nu}\right)}{\cos \alpha_{j}-\cos \alpha_{\nu}} \\
& =-\frac{1}{2 N} \sum_{\nu=1}^{N} \frac{\gamma\left(\alpha_{\nu}\right) \sin \alpha_{\nu}}{\cos \alpha_{j}-\cos \alpha_{\nu}} .
\end{aligned}
$$

As shown in the above, chordwise vortex distribution is treated as continuous in QCM, but the resulting numerical formula Eq. (10) looks like that of VLM in which discrete and concentrated vortex distribution is used. In the case of a twodimensional wing, vortex distribution $\gamma(\alpha)$ obtained by QCM agrees very well with analytical solution.

As for three-dimensional wing, the characteristics of QCM can be summarized as follows;

(1) Continuous vortex distribution over a wing is replaced with a quasi-continuous one, i.e. continuous in chordwise direction and stepwise constant in spanwise direction. In the surface integral over a chordwise vortex strip, spanwise integration is carried out analytically, while chordwise integration is carried out numerically by the midpoint trapezoidal rule. The square root singularity at leading edge and the Cauchy singularity are properly accounted for as mentioned above.

(2) Loading points (integration points) and control points over a wing are arranged according to the semi-circle method as shown in Fig. 1. Chordwise arrangement is chosen so as to eliminate the Cauchy singularity. Since control points are placed on trailing edge, Kutta condition can be satisfied automatically, i.e. the flow is tangent to wing at the trailing edge. In the spanwise direction, finer vortex strips are used toward wing tip where sectional properties vary rapidly. Outermost loading stations do not coincide with wing tips and it contributes to the quick convergence of calculation ${ }^{15)}$.

(3) Accuracy in calculation is satisfactory. As an example, the results calculated for a planar circular wing are shown in comparison with analytical solution ${ }^{16)}$ in Fig. 2. Spanwise lift distribution and chordwise loading distributions at center $(\eta=0.0)$ and at quarter-span $(\eta=0.50)$ sections obtained by QCM agree very well with analy- 




Spanw18e Arrangement
- loading Point

- Control Point

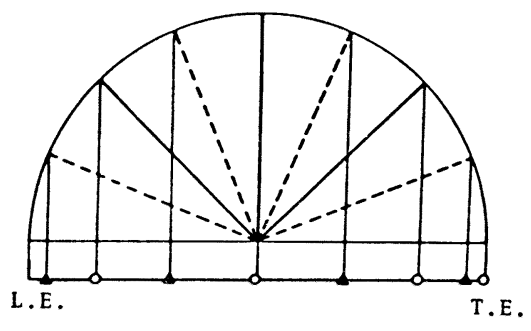

Chordwise Arrangement

Fig. 1 Arrangement of loading and control points over a wing
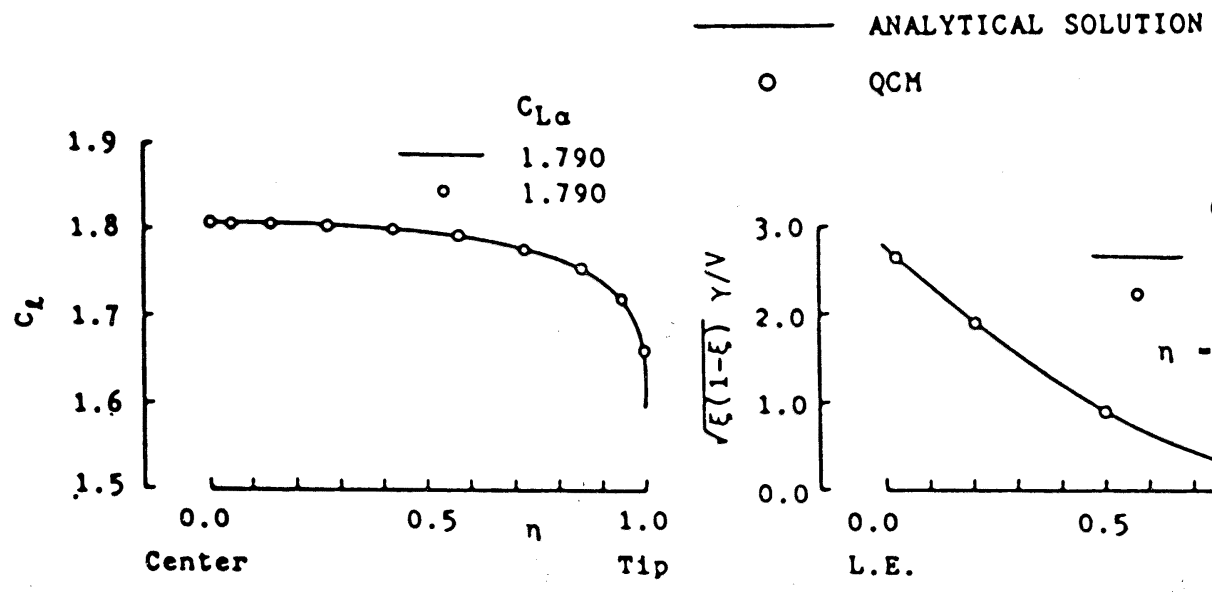

$C_{l}$
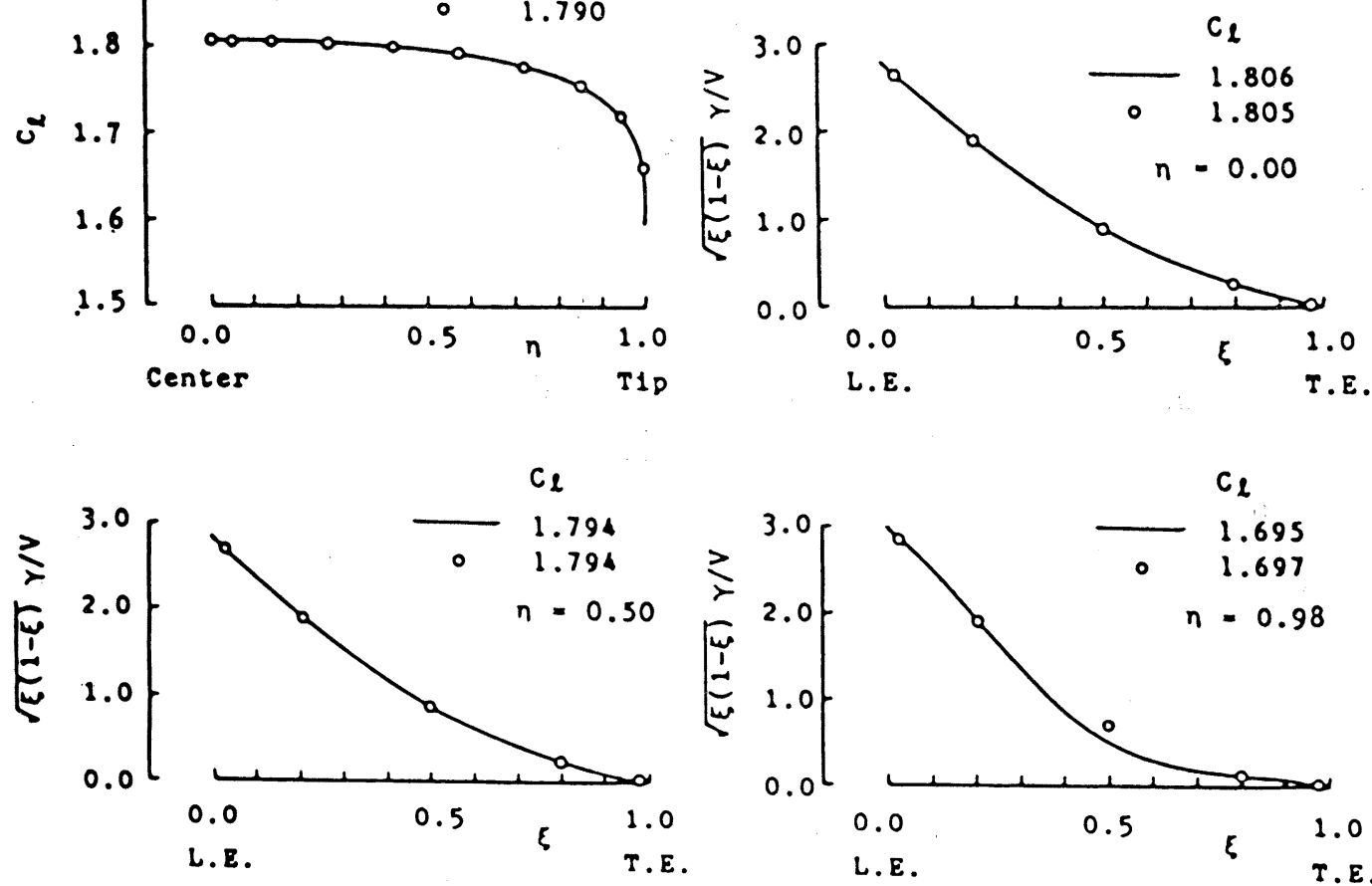

Fig. 2 Spanwise lift and chordwise loading distributions for a planar circular wing

tical solution. Sufficient accuracy is retained also in the neighbourhood of wing tip $(\eta=0.98)$. Geometry of the propeller blade in tip region is similar to that of the circular wing. Then accurate solution can be expected in the neighbourhood of the propeller blade tip.

(4) Quick convergence is attained. Fig. 3 shows the results calculated for a planar rec- tangular wing of aspect ratio 2.0. The numbers of spanwise control points $M$ and chordwise control points $N$ were varied systematically. Converged solution is obtained with a small number of control points. 

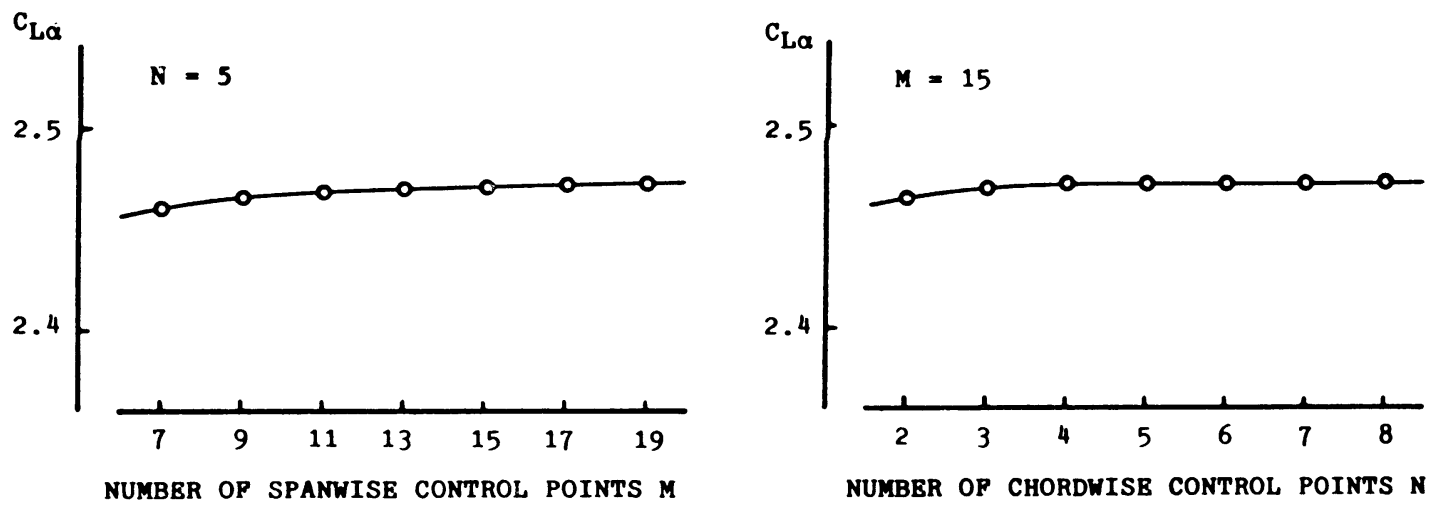

Fig. 3 Lift coefficient for a planar rectangular wing of aspect ratio 2.0

\section{A Numerical Method to Estimate Propeller Open-Water Characteristics Based on Quasi-Continuous Method}

\subsection{Coordinate Systems and Geometry of Propeller}

Let us consider a propeller rotating clockwise with the angular velocity $\Omega$ in a uniform flow with the velocity $V_{A}$ of an unbounded, incompressible and inviscid fluid. The propeller consists of a set of axisymmetrically arranged blades of identical shape. The blades are assumed to be thin and the presence of propeller boss is neglected.

For geometrical representation of a propeller, we define a rectangular coordinate system O-xyz fixed on the propeller as shown in Fig. 4. The $x$ axis is chosen so as to coincide with the axis of revolution of propeller with the origin $\mathrm{O}$ at an appropriate point on the axis. The positive direction of the $x$-axis is taken in the downstream. The $z$-axis coincides with the generator line of the first blade and is positive outward from the origin $\mathrm{O}$. The $y$-axis completes the right handed coordinate system.

A cylindrical coordinate system is defined as follows. Angular coordinate $\theta$ is measured clockwise from the $z$-axis when viewed in the direction of positive $x$. Radial and angular coordinates are given by

$$
r=\sqrt{y^{2}+z^{2}}, \quad \theta=\tan ^{-1}(-y / z) .
$$

Then, the rectangular coordinate system O-xyz is transformed into the cylindrical coordinate system O- $x r \theta$ by the relation

$$
x=x, \quad y=-r \sin \theta, \quad z=r \cos \theta .
$$

Here we introduce a chordwise coordinate s along the nose-tail helix at each blade section and the positive direction is taken from leading to

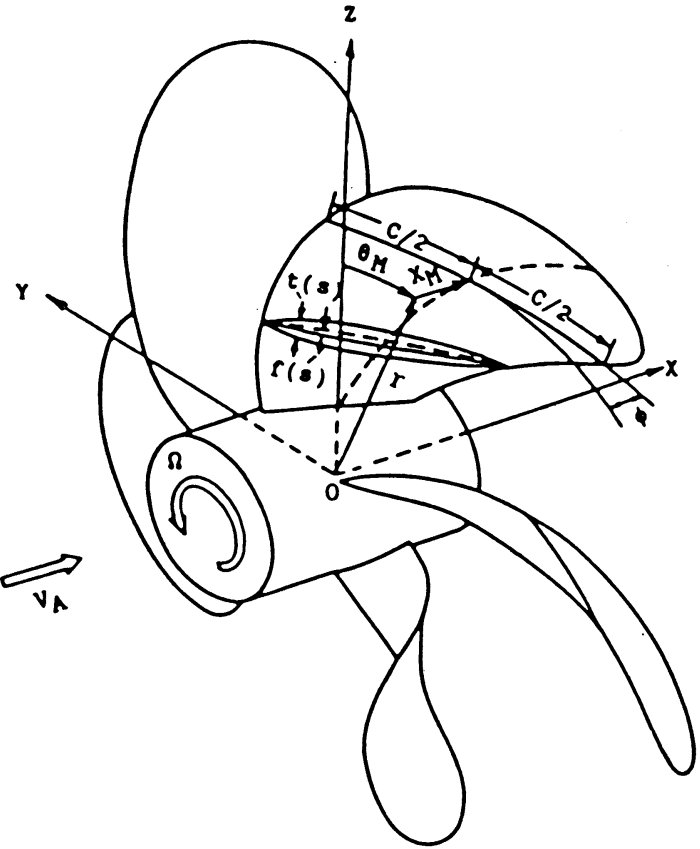

Fig. 4 Coordinate systems and blade geometry

trailing edges. Let the $s$-coordinates of leading and trailing edges be $s_{L}(v)$ and $s_{T}(r)$ respectively, then the chord length $c(v)$ at radius $r$ is expressed by

$$
c(r)=s_{T}(v)-s_{L}(v) .
$$

Skew angle $\theta_{\boldsymbol{M}}(v)$ and rake $x_{\boldsymbol{M}}(v)$ are defined as the angular coordinate and $x$-coordinate of midchord line respectively, as shown in Fig. 4. Denoting the pitch angle of nose-tail helix by $\phi(r)$, the coordinates of leading and trailing edges are given by 


$$
\begin{aligned}
& x_{L, T}(r)=x_{M}(r) \mp \frac{1}{2} c(r) \sin \phi(r) \\
& \theta_{L, T}(r)=\theta_{M}(r) \mp \frac{1}{2} \frac{c(r)}{r} \cos \phi(r) \\
& y_{L, T}(r)=-r \sin \theta_{L, T}(r) \\
& z_{L, T}(r)=r \cos \theta_{L, T}(r) .
\end{aligned}
$$

where the subscripts $L$ and $T$ stand for leading and trailing edges respectively.

Camber and thickness of blade section are defined in a way similar to that of a two-dimensional section by the functions $f(r, s)$ and $t(r, s)$ respectively. The camber $f(r, s)$ is measured in the cylindrical plane of radius $r$ at right angle to nose-tail helix and the thickness $t(r, s)$ is added symmetrically and at right angle to the camber $f(r, s)$.

Here we introduce a non-dimensional chordwise coordinate $\xi$, which is zero at leading edge and unity at trailing edge. $\xi$ is related to $s$ by the relation

$$
s(r)=s_{L}(r)+c(v) \xi .
$$

Then the coordinate of a point on the mean camber surface of the $k$-th blade can be written as

$$
\begin{aligned}
x_{c}(r, \xi)= & x_{M}(r)+c(r)\left(\xi-\frac{1}{2}\right) \sin \phi(r) \\
& -f(r, \xi) \cos \phi(r) \\
\theta_{c}(r, \xi)= & \theta_{M}(r)+c(r)\left(\xi-\frac{1}{2}\right) \frac{\cos \phi(r)}{r} \\
& +f(r, \xi) \frac{\sin \phi(r)}{r}+\theta_{k} \\
y_{c}(r, \xi)= & -r \sin \theta_{c}(r, \xi) \\
z_{c}(r, \xi)= & r \cos \theta_{c}(r, \xi)
\end{aligned}
$$

where

$$
\begin{gathered}
\theta_{k}=\frac{2 \pi(k-1)}{K}, \quad k=1,2, \cdots \cdots, K, \\
K=\text { number of blades }
\end{gathered}
$$

and the subscript $C$ denotes mean camber surface.

3.2 Formulation of Numerical Model of Propeller Lifting Surface

Propeller blades are represented by the distributions of vortices and sources on the mean camber surfaces of blades together with the associated distribution of vortices shed into the wake. The vortex system, i.e. the distribution of horseshoe vortices which consist of bound vortices and free vortices shed from both edges of bound vortices, represents blade loading and wake, and the source distribution represents blade thickness.

Continuous distributions of bound vortices and sources are replaced with quasi-continuous ones according to QCM. Thus the blades are covered with a number of vortex strips with associated free vortices and source strips.
Loading points and control points are arranged on the mean camber surface of blade according to QCM. In the radial direction, loading stations (radial coordinates of the edges of each vortex/ source strip) and control stations (radial coordinates of control points) are chosen at the radii defined as follows. For loading stations

$$
\begin{array}{r}
r_{L_{\mu}}=\frac{1}{2}\left(r_{0}+r_{B}\right)-\frac{1}{2}\left(r_{0}-r_{B}\right) \cos \beta_{\mu}, \\
\mu=1,2, \cdots \cdots, M+1,
\end{array}
$$

and for control stations

$$
\begin{array}{r}
r_{c i}=\frac{1}{2}\left(r_{0}+r_{B}\right)-\frac{1}{2}\left(r_{0}-r_{B}\right) \cos \beta_{i}, \\
i=1,2, \cdots \cdots, M,
\end{array}
$$

where

$$
\begin{aligned}
& r_{0}=\text { propeller radius, } \\
& r_{B}=\text { boss radius, } \\
& M=\text { number of radial control points, } \\
& \beta_{\mu}=\frac{2 \mu-1}{2(M+1)} \pi, \quad(\mu=1,2, \cdots \cdots, M+1), \\
& \beta_{i}=\frac{i}{M+1} \pi, \quad(i=1,2, \cdots \cdots, M) .
\end{aligned}
$$

At the loading and control stations, chordwise loading and control points are arranged according to QCM, i.e. for loading points

$$
\begin{array}{r}
s_{\mu \nu}=S_{L}\left(r_{L_{\mu}}\right)+\frac{1}{2} c\left(\gamma_{L_{\mu}}\right)\left(1-\cos \alpha_{\nu}\right), \\
\nu=1,2, \cdots \cdots, N,
\end{array}
$$

and for control points

$$
\begin{aligned}
s_{i j}=s_{L}\left(r_{c i}\right)+\frac{1}{2} c\left(v_{c i}\right) & \left(1-\cos \alpha_{j}\right), \\
j & =1,2, \cdots \cdots, N,
\end{aligned}
$$

where

$$
\begin{aligned}
& N=\text { number of chordwise control points, } \\
& \alpha_{\nu}=\frac{(2 \nu-1)}{2 N} \pi, \quad(\nu=1,2, \cdots \cdots, N), \\
& \alpha_{j}=\frac{j}{N} \pi, \quad(j=1,2, \cdots \cdots, N) .
\end{aligned}
$$

Then, loading points and control points are arranged on the same fraction-chord lines respectively. We call these lines as loading lines and control lines respectively, in this paper.

An example of the arrangement of loading lines and loading stations with control points is shown in Fig. 5. Free vortices are shed only from the loading stations due to the stepwise distribution of vortices. The trailing vortex sheet (free vortex sheet in the wake) is, therefore, composed of $(M+$ 1) helical vortices.

\subsection{Calculation of Induced Velocities}

Induced velocity at a control point $P_{i j}$ due to 

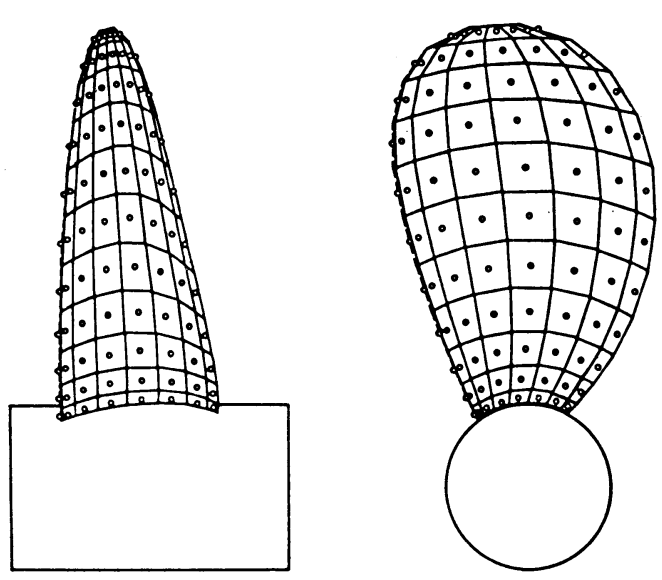

Fig. 5 Arrangement of loading lines, loading stations and control points
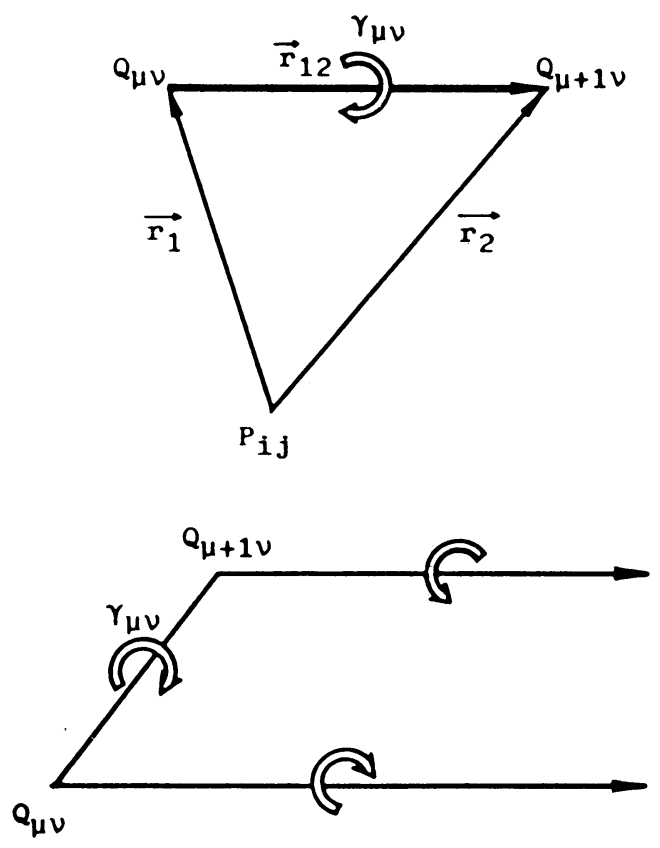

Fig. 6 Vortex segment and horseshoe vortex

a vortex segment of unit strength placed between loading points $Q_{\mu \nu}$ and $Q_{\mu+1 \nu}$ (see Fig. 6) is given by Biot-Savart law ${ }^{13)}$ as

$$
\vec{v}_{i j \mu \nu}^{V}=\frac{1}{4 \pi} \frac{\vec{r}_{1} \times \vec{r}_{12}}{\left|\overrightarrow{r_{1}} \times \vec{r}_{12}\right|^{2}}\left\{\frac{\vec{r}_{2}}{\left|\overrightarrow{r_{2}}\right|}-\frac{\vec{r}_{1}}{\left|\overrightarrow{r_{1}}\right|}\right\} \cdot \vec{r}_{12} .
$$

Here we consider a horseshoe vortex of unit strength, which consists of bound vortex placed at $Q_{\mu \nu}$ and $Q_{\mu+1 \nu}$ and two free vortices shed from $Q_{\mu \nu}$ and $Q_{\mu+1 \nu}$ in the chordwise direction along loading stations (see Fig. 6). Induced velocity at a control point $P_{i j}$ due to this horseshoe vortex is expressed by

$$
\begin{aligned}
\vec{v}_{i j \mu \nu}^{G}= & \left.\vec{v}_{i j \mu \nu}^{B}+\sum_{\nu^{\prime}=\nu}^{N} \overrightarrow{(v}_{i j \mu+1 \nu^{\prime}}^{F}-\vec{v}_{i j \mu \nu^{\prime}}^{F}\right) \\
& +\vec{v}_{i j \mu+1}^{T}-\vec{v}_{i j \mu}^{T}
\end{aligned}
$$

where

$\vec{v}_{i j_{\mu \nu}}^{B}=$ induced velocity at $P_{i j}$ due to vortex segment (bound vortex) of unit strength placed between $Q_{\mu \nu}$ and $Q_{\mu+1 \nu,}$

$\vec{v}_{i j \mu \nu}^{F}=$ induced velocity at $P_{i j}$ due to vortex segment (free vortex) of unit strength placed between $Q_{\mu \nu}$ and $Q_{\mu \nu+1}$,

$\vec{v}_{i j_{\mu}}^{T}=$ induced velocity at $P_{i j}$ due to trailing vortex of unit strength shed from trailing edge at loading station $\gamma=r_{L \mu}$,

$\vec{v}_{i j \mu \nu}^{B}$ and $\vec{v}_{i j \mu \nu}^{F}$ can be calculated by Eq. (20) and $\vec{v}_{i j_{\mu}}^{T}$ also can be calculated with Eq. (20) by replacing helical vortex line with vortex segments of appropriate length.

Let the strength of bound vortices in the $\mu$-th vortex strip be $\gamma_{\mu}(s)$, then induced velocity at $P_{i j}$ due to horseshoe vortices on the vortex strip is expressed based on the procedure described in the previous section 2 , as

$$
\begin{aligned}
\vec{V}_{i j_{\mu}}^{G} & =\int_{s_{L}}^{s T} \gamma_{\mu}\left(s^{\prime}\right) \vec{v}_{i j_{\mu}}^{G}\left(s^{\prime}\right) d s^{\prime} \\
& =\frac{c_{\mu}}{2} \int_{0}^{\pi} \gamma_{\mu}\left(\alpha^{\prime}\right) \vec{v}_{i j_{\mu}}^{G}\left(\alpha^{\prime}\right) \cdot \sin \alpha^{\prime} d \alpha^{\prime} \\
& \cong \frac{\pi c_{\mu}}{2 N} \sum_{\nu=1}^{N} \gamma_{\mu}\left(\alpha_{\nu}\right) \vec{v}_{i j_{\mu}}^{G}\left(\alpha_{\nu}\right) \sin \alpha_{\nu} \\
& =\frac{\pi c_{\mu}}{2 N} \sum_{\mu=1}^{N} \gamma_{\mu \nu} \vec{v}_{i j_{\mu \nu}}^{G} \sin \alpha_{\nu}
\end{aligned}
$$

where

$$
c_{\mu}=c\left(v_{c_{\mu}}\right), \quad \gamma_{\mu \nu}=\gamma_{\mu}\left(\alpha_{\nu}\right), \quad \vec{v}_{i j^{\prime \nu}}^{G}=\vec{v}_{i j^{\prime \prime}}^{G}\left(\alpha_{\nu}\right) .
$$

The induced velocity at a control point $P_{i j}$ due to whole vortex system is obtained by the next equation

$$
\begin{aligned}
\vec{V}_{i j}^{G} & =\sum_{k=1}^{K} \sum_{\mu=1}^{M} \vec{V}_{i \mu_{\mu} k}^{G} \\
& =\frac{\pi}{2 N} \sum_{k=1}^{K} \sum_{\mu=1}^{M} c_{\mu} \sum_{\nu=1}^{N} \gamma_{\mu \nu} \vec{v}_{i j \mu \nu k}^{G} \sin \alpha_{\nu}
\end{aligned}
$$

where the suffix $k$ shows the contribution from the $k$-th blade.

Next let us consider the induced velocity due to the source distribution. Induced velocity at a control point $P_{i j}$ due to a source segment of unit strength placed between loading points $Q_{\mu \nu}$ and $Q_{\mu+1 \nu}$ (see Fig. 6) is given in a similar manner to that of vortex segment by 


$$
\vec{v}_{i j \mu \nu}^{S}=\frac{1}{4 \pi} \frac{\vec{r}_{1} \times \vec{r}_{12}}{\left|\vec{r}_{1} \times \vec{r}_{12}\right|^{2}} \times\left\{\frac{\vec{r}_{2}}{\left|\overrightarrow{r_{2}}\right|}-\frac{\vec{r}_{1}}{\left|\vec{r}_{1}\right|}\right\}\left|\vec{r}_{12}\right| \text {. }
$$

Denoting the source strength at the $\nu$-th loading point in the $\mu$-th source strip by $\sigma_{\mu \nu}$, the induced velocity at a control point $P_{i j}$ due to whole source distribution is expressed as

$$
\vec{V}_{i j}^{S}=\frac{\pi}{2 N} \sum_{k=1}^{K} \sum_{\mu=1}^{M} c_{\mu} \sum_{\nu=1}^{N} \sigma_{\mu \nu} \vec{v}_{i j \mu \nu k}^{S} \sin \alpha_{\nu} .
$$

\subsection{Boundary Condition}

Since we are treating steady problems, all the blades are in the same condition. Then the boundary condition is that the velocity normal to the blade mean camber surface is zero at the control points on the first blade. Thus the next equations hold at the control points $P_{i j}$,

$$
\begin{aligned}
& \left(\vec{V}_{i j}^{G}+\vec{V}_{i j}^{S}+\vec{V}_{i j}^{I}\right) \cdot \vec{n}_{i j}=0, \\
& \quad i=1,2, \cdots \cdots, M, \quad j=1,2, \cdots \cdots, N,
\end{aligned}
$$

where

$\vec{V}_{i j}^{G}=$ induced velocity due to vortex system,

$\vec{V}_{i j}^{S}=$ induced velocity due to source distribution,

$\vec{V}_{i j}^{I}=$ relative inflow velocity,

$\vec{n}_{i j}=$ unit vector normal to blade camber surface and the positive direction is taken from face to back.

The unit normal vector $\vec{n}_{i j}$ is obtained from the cross product of two diagonal vectors connecting corners of each quadrilateral element composed of loading lines and loading stations. In this element control point $P_{i j}$ is located (see Fig. 5).

The strength of source distribution $\sigma$ representing blade thickness can be expressed approximately by thin wing theory as the quantity proportional to the product of chordwise derivative of thickness form and relative inflow velocity. In the present method, source strength thus obtained is used.

Then the boundary condition Eq. (26) is now reduced to linear simultaneous equations with respect to the unknown vortex strength $\gamma_{\mu \nu}$. Eq. (26) is now rewritten as

$$
\begin{aligned}
& \sum_{\mu=1}^{M} \sum_{\nu=1}^{N} A_{i j \mu \nu} \gamma_{\mu \nu}=-\vec{n}_{i j} \cdot\left(\vec{V}_{i j}^{S}+\vec{V}_{i j}^{I}\right), \\
& \quad i=1,2, \cdots \cdots, M, \quad j=1,2, \cdots \cdots, N,
\end{aligned}
$$

where

$$
\begin{aligned}
A_{i j_{\mu \nu}=} & \text { influence function, i.e., normal velocity } \\
& \text { at the }(i, j) \text {-th control point induced by } \\
& \text { the }(\mu, \nu) \text {-th horseshoe vortices of unit } \\
& \text { strength on all the blades. }
\end{aligned}
$$

The vortex strength $\gamma_{\mu \nu}$ can be obtained by solving Eq. (27).

\subsection{Calculation of Thrust, Torque and Pressure Distribution}

\section{(1) Potential Component}

Forces acting on a vortex/source segment placed between $Q_{\mu \nu}$ and $Q_{\mu+1 \nu}$ can be obtained from Kutta-Joukowski theorem and Lagally's theorem as

$$
\vec{F}_{\mu \nu}=\rho \vec{V}_{\mu \nu} \times \vec{\gamma}_{\mu \nu}-\rho \vec{V}_{\mu \nu} \sigma_{\mu \nu}\left|\vec{l}_{\mu \nu}\right|
$$

where

$$
\begin{aligned}
\vec{V}_{\mu \nu}= & \vec{V}_{\mu \nu}^{G}+\vec{V}_{\mu \nu}^{S}+\vec{V}_{\mu \nu}^{I} \\
= & \text { resultant flow velocity at the midpoint } \\
& \text { of segment } \\
\vec{\gamma}_{\mu \nu}= & \gamma_{\mu \nu} \vec{\nu}_{\mu \nu} \\
= & \text { vortex segment vector } \\
\vec{l}_{\mu \nu}= & \text { segment vector } \vec{Q}_{\mu \nu} \vec{Q}_{\mu+1 \nu} \\
\rho= & \text { fluid density }
\end{aligned}
$$

Then, thrust and torque acting on whole propeller are obtained by following equations,

$$
\begin{aligned}
T_{0}= & -K \int_{r_{B}}^{r_{0}} d r^{\prime} \int_{s_{L}}^{s_{T}} F_{x} d s^{\prime} \\
= & -K \sum_{\mu=1}^{M} \frac{\pi c_{\mu}^{*}}{2 N} \sum_{\nu=1}^{N}\left(F_{x}\right)_{\mu \nu} \sin \alpha_{\nu} \\
Q_{0}= & K \int_{r_{B}}^{r_{0}} d r^{\prime} \int_{s_{L}}^{s_{T}}\left(-F_{y} \cdot z+F_{z} \cdot y\right) d s^{\prime} \\
= & K \sum_{\mu=1}^{M} \frac{\pi c_{\mu}^{*}}{2 N} \sum_{\nu=1}^{N}\left[-\left(F_{y}\right)_{\mu \nu} z_{\mu \nu}\right. \\
& \left.+\left(F_{z}\right)_{\mu \nu} y_{\mu \nu}\right] \sin \alpha_{\nu},
\end{aligned}
$$

where

$\left(F_{x}\right)_{\mu \nu}, \quad\left(F_{y}\right)_{\mu \nu}, \quad\left(F_{z}\right)_{\mu \nu}=x-, y$ - and $z$ - components of $\vec{F}_{\mu \nu}, x_{\mu \nu}, y_{\mu \nu}, z_{\mu \nu}=x-, y$ - and $z$ - coordinates at the midpoint of the segment, $C_{\mu}^{*}=$ chord length at $r=1 / 2\left(\gamma_{L_{\mu}}+\gamma_{L_{\mu+1}}\right)$.

In addition to the forces acting on vortex and source distributions mentioned in the above, leading edge suction force must be taken into consideration. The leading edge suction force results from leading edge singularity of vortex distribution under thin wing assumption. The suction force per unit span is given by

$$
F_{s}=\frac{1}{4} \pi \rho c C_{s^{2}}
$$

where

$$
C_{s}=\lim _{s \rightarrow s_{L}} \gamma(s) \sqrt{\frac{s-s L}{c}}
$$

and the coefficient $C_{s}$ can be obtained directly in $\mathrm{QCM}^{13}$ ) then, thrust and torque resulting from leading edge suction force are expressed as 


$$
\begin{aligned}
T_{s} & =K \int_{r_{B}}^{r_{0}} F_{s}\left(r^{\prime}\right) \sin \phi\left(r^{\prime}\right) d r^{\prime} \\
& =K \frac{\pi\left(r_{0}-r_{B}\right)}{2 M} \sum_{i=1}^{M} F_{\boldsymbol{s}}\left(r_{c i}\right) \sin \phi\left(r_{c i}\right) \sin \beta_{i} \\
Q_{s} & =-K \int_{r_{B}}^{r_{0}} F_{\boldsymbol{s}}\left(r^{\prime}\right) \cos \phi\left(r^{\prime}\right) r^{\prime} d r^{\prime} \\
& =-K \frac{\pi\left(r_{0}-r_{B}\right)}{2 M} \sum_{i=1}^{M} F_{\boldsymbol{s}}\left(r_{c i}\right) \cos \phi\left(r_{c i}\right) r_{c i} \sin \beta_{i}
\end{aligned}
$$

(2) Viscous Component

Let the viscous drag coefficient of blade element at the radius $r$ be $C_{D}(r)$, then thrust and torque due to viscous drag are expressed as

$$
\begin{aligned}
T_{D}= & -K \frac{1}{2} \rho \int_{r_{B}}^{r_{0}} C_{D}\left(r^{\prime}\right) \bar{W}\left(r^{\prime}\right) \bar{V}_{x}\left(r^{\prime}\right) c\left(r^{\prime}\right) d r^{\prime} \\
= & -K \rho \frac{\pi\left(r_{0}-r_{B}\right)}{4 M} \sum_{i=1}^{M} C_{D}\left(r_{c i}\right) \bar{W}\left(r_{c i}\right) \\
& \cdot \bar{V}_{x}\left(r_{c i}\right) c\left(r_{c i}\right) \sin \beta_{i} \\
Q_{D}= & K \frac{1}{2} \rho \int_{r_{B}}^{r_{0}} C_{D}\left(r^{\prime}\right) \bar{W}\left(r^{\prime}\right) \bar{V}_{\theta}\left(r^{\prime}\right) c\left(r^{\prime}\right) r^{\prime} d r^{\prime} \\
= & K \rho \frac{\pi\left(r_{0}-r_{B}\right)}{4 M} \sum_{i=1}^{M} C_{D}\left(r_{c i}\right) \bar{W}\left(r_{c i}\right) \\
& \cdot \bar{V}_{\theta}\left(r_{c i}\right) c\left(r_{c i}\right) r_{c i} \sin \beta_{i}
\end{aligned}
$$

where

$\bar{W}(r)=\sqrt{\overline{\bar{V}_{x}(r)^{2}+\bar{V}_{\theta}(r)^{2}}}$,

$\bar{V}_{x}(r)=x$-component of resultant flow velocity averaged in the chordwise direction,

$\bar{V}_{\theta}(r)=\theta$-component of resultant flow velocity averaged in the chordwise direction.

The total thrust and torque are given by

$$
\left.\begin{array}{l}
T=T_{0}+T_{S}+T_{D} \\
Q=Q_{0}+Q_{S}+Q_{D}
\end{array}\right\}
$$

The thrust coefficient $K_{T}$, torque coefficient $K_{Q}$ and propeller efficiency $e P$ are defined by

$$
K_{T}=\frac{T}{\rho n^{2} D^{4}}, \quad K_{Q}=\frac{Q}{\rho n^{2} D^{5}}, \quad e P=\frac{J}{2 \pi} \cdot \frac{K_{T}}{K_{Q}},
$$

where

$D=$ propeller diameter,

$n=$ propeller revolutions per unit time,

$J=V_{A} / n D:$ advance coefficient.

(3) Pressure distribution

The resultant flow velocities on back and face at the control points $P_{i j}$ on the mean camber surface are expressed as

$$
\vec{V}_{i j \pm}=\vec{V}_{i j}^{G}+\vec{V}_{i j}^{S}+\vec{V}_{i j}^{I} \pm \frac{1}{2}\left(\gamma_{i j} \cdot \vec{\tau}_{i j}+\sigma_{i j} \cdot \vec{n}_{i j}\right),
$$

where

$\gamma_{i j}=$ vortex strength at $P_{i j}$,

$\sigma_{i j}=$ source strength at $P_{i j}$,

$\vec{\tau}_{i j}=$ unit chordwise tangent vector at $P_{i j}$.

Subscripts \pm denote back (suction side) and face (pressure side) respectively. The pressure coefficients at point $P_{i j}$ on back and face are expressed by Bernoulli's theorem as

$$
\begin{aligned}
C_{P_{i j \pm}} & =\frac{P_{i j_{ \pm}}-P_{0}}{\frac{1}{2} \rho W_{0}^{2}} \\
& =1-\frac{\left|\vec{V}_{i j_{ \pm}}\right|^{2}}{W_{0}^{2}},
\end{aligned}
$$

where

$$
\begin{aligned}
& P_{0}=\text { ambient pressure in uniform flow, } \\
& W_{0}=\sqrt{V_{A}^{2}+\left(v_{c i} \Omega\right)^{2}} .
\end{aligned}
$$

\section{Practical Method for Estimation of Open-Water Characteristics and Pressure Distribution on Blade}

In the practical calculation, the model of trailing vortex wake is formulated as follows. Trailing vortices leave the trailing edge in the direction tangent to the mean camber surfaces so as to satisfy Kutta condition. After that the pitch of trailing vortices changes linearly with respect to angular coordinate $\theta$ and reaches an ultimate value within a half revolution. This ultimate value is defined as the mean of geometrical pitch distribution of the propeller blade. In the downstream after that, the trailing vortices become helical lines of constant pitch. Further the contraction of wake is neglected in the present calculation. This wake model was chosen after some preliminary studies and it was found that this wake model gives good correlation with experiments in the slope of thrust and torque coefficients against advance coefficient in wide range.

The propeller performances can be calculated by the method mentioned in the previous section 3 , provided the sectional drag coefficient $C_{D}$ is given. In reality, however, some correction is necessary to correlate calculated results with experiments. In the present method, an empirical correction factor $C_{V}$ is introduced in the right hand side of Eq. (27) as follows,

$$
\begin{array}{r}
\sum_{\mu=1}^{M} \sum_{\nu=1}^{N} A_{i j \mu \nu} \gamma_{\mu \nu}=-\left[\vec{n}_{i j} \cdot\left(\vec{V}_{i j}^{S}+\vec{V}_{i j}^{I}\right)\right] \cdot C_{V i}, \\
i=1,2, \cdots \cdots, M, j=1,2, \cdots \cdots, N,
\end{array}
$$

where

$\left.C_{V i}=0.97-2.15 t / c\right)_{i}$,

$t / c)_{i}=$ blade thickness to chord ratio at the control station $r=r_{c i}$. 
Eq. (38) was introduced based on the preliminary calculations made on methodical series propellers. This correction factor implies some reduction in the angle of attack of relative inflow to blade section. This correction is similar to that used in the method of Kerwin and Lee ${ }^{9)}$ in which the geometrical pitch angle of blade section is reduced by some amount. Further in the present paper, the components of thrust and torque resulting from the force acting on the source distribution and leading edge suction force are neglected assuming as small quantities. Therefore, the effects of these components are practically included in the correction factor $C_{V}$.

As for sectional drag coefficient, the following formula was applied referring to NACA airfoil section data ${ }^{17}$,

$$
\left.\begin{array}{ll}
C_{D}=C_{D \min }, & \left(C_{l}<0.2\right) \\
C_{D}=C_{D \min }+0.03\left(C_{l}-0.2\right) & \left(C_{l} \geqq 0.2\right)
\end{array}\right\}
$$

where $C_{l}=$ sectional lift coefficient. For minimum profile drag coefficient $C_{D \text { min }}$, the following formula was adopted.

$$
C_{D \min }=0.012 t / c+2(1+2 t / c) C_{f(\text { Hughes })}
$$
where

$C_{f_{\text {(Hughes })}}=0.066 /\left(\log _{10} R_{n}-2.03\right)^{2}$,

$R_{n}=\left(W_{0} \times C\right) / \nu$, Reynolds number at each blade section.

Eq. (40) was obtained from the resistance tests on a wing section with chord length of $3.5 \mathrm{~m}^{18)}$. This expression is applicable to the estimation of minimum profile drag coefficient for fully turbulent flow condition. Torque coefficients calculated with Eqs. (39) and (40) were, however, a little higher than experimental data for model propellers. The discrepancy seemed to result from the fact that laminar boundary layer still remains in the case of model propellers ${ }^{19}$. Then the next formula for model propellers of ordinary size $(D=$ $200 \mathrm{~mm}-300 \mathrm{~mm}$ ) was introduced empirically as the minimum drag coefficient,

$$
C_{D \min }=0.0084+0.16 t / c \text {. }
$$

\section{Numerical Examples}

\subsection{Propeller Open-Water Characteristics}

To evaluate the applicability of the present method, the propeller open-water characteristics were calculated for the following six propellers with variation of skew and pitch distributions, and compared with published experimental data. In these calculations, the number of control points was chosen to be 11 in radial and 8 in chordwise directions.

\section{SR183 Propeller MP 218 (Conv. Prop.)}

SR183 Propeller MP 220 ( 45 deg. skew)
DTNSRDC Propeller 4381

DTNSRDC Propeller 4382

DTNSREC Propeller 4383

( 0 deg. skew)

( 36 deg. skew)

( 72 deg. skew)

DTNSRDC Propeller 4384

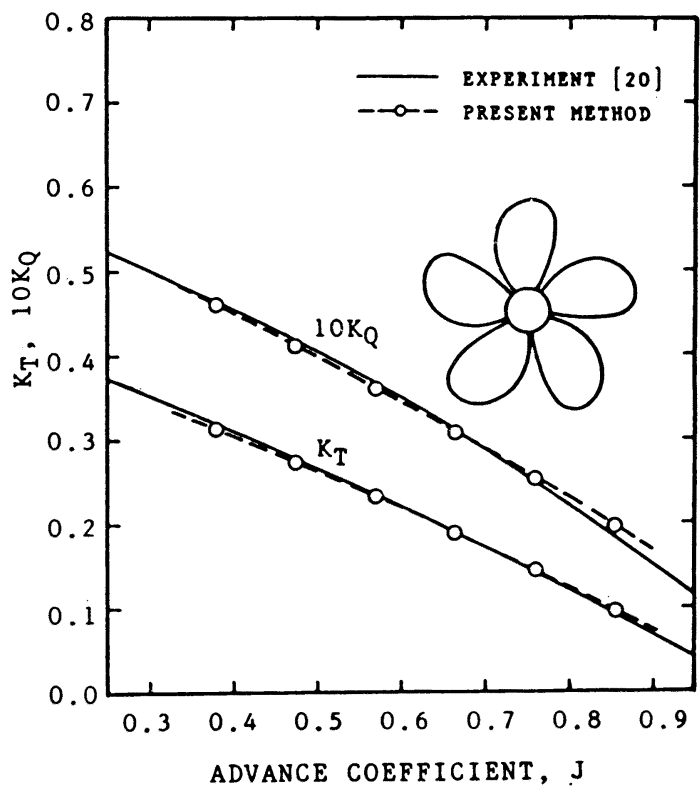

Fig. 7 Comparison of measured and calculated open-water characteristics of SR 183 Propeller MP 218 (Conv. Prop.)

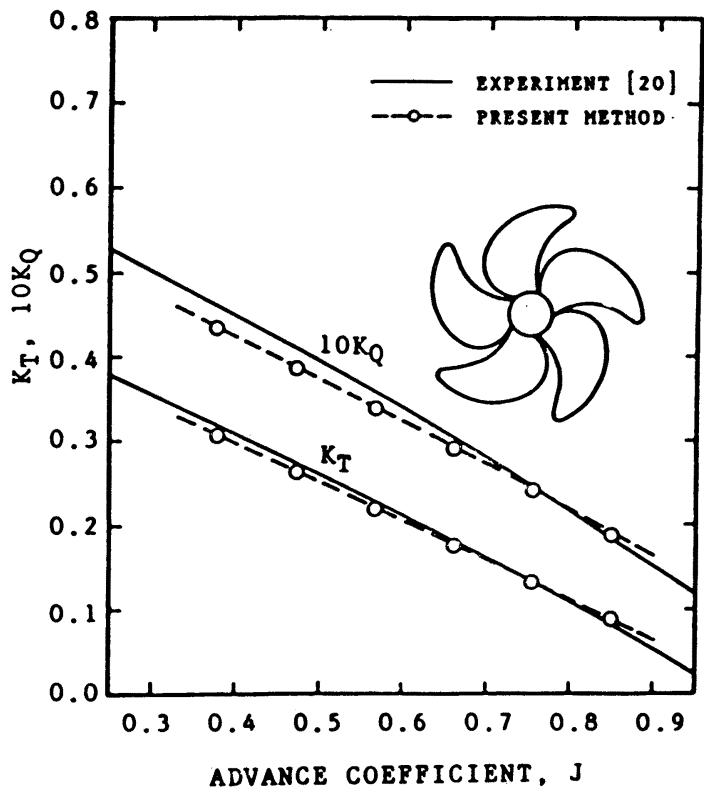

Fig. 8 Comparison of measured and calculated open-water characteristics of SR 183 Propeller MP 220 (45-deg skew) 


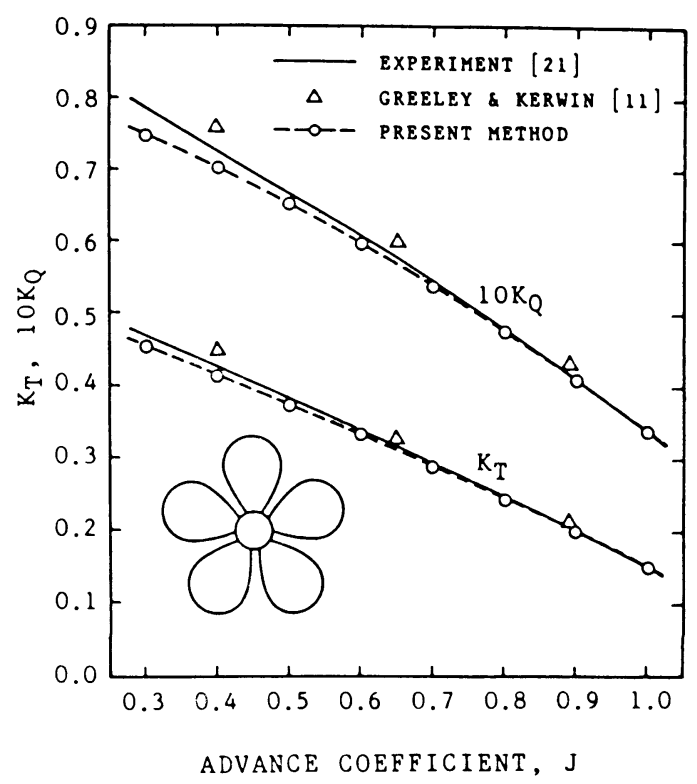

Fig. 9 Comparison of measured and calculated open-water characteristics of DTNSRDC Propeller 4381 (0-deg skew)

SR183 propellers MP 218 and MP 220 are the conventional propeller and the highly skewed propeller designed for SEIUN MARU ${ }^{20)}$. Figs. 7 and 8 show the open-water characteristics calculated by the present method in comparison with the measured data. In the case of the Propeller MP 218, agreement is good both for thrust coefficient $K_{T}$ and torque coefficient $K_{Q}$. As for the Propeller MP 220, $K_{T}$ agrees fairly well, but the calculated $K_{Q}$ is a little lower than the experiment.

DTNSRDC Propellers 4381, 4382, 4383 and 4384 are the five bladed skew series propellers ${ }^{21}$. Comparisons of measured and calculated openwater characteristics are shown in Figs. 9, 10, 11 and 12 , together with the results calculated by Greeley and Kerwin ${ }^{11)}$ for reference. As for the Propellers 4381, 4382 and 4383, calculated $K_{T}$ and $K_{Q}$ by the present method agree fairly well with experimental data. But in the case of the Propeller 4384, both of the calculated $K_{T}$ and $K_{Q}$ are higher than experimental data. Comparing with the calculated data by Greeley and Kerwin, the present method seems to give a little closer value to experimental data. Judging from the above results, the accuracy of the present method seems to be pretty good, but the present method still cannot predict the open-water characteristics of the propellers with extremely large skew such as DTNSRDC Propeller 4384.

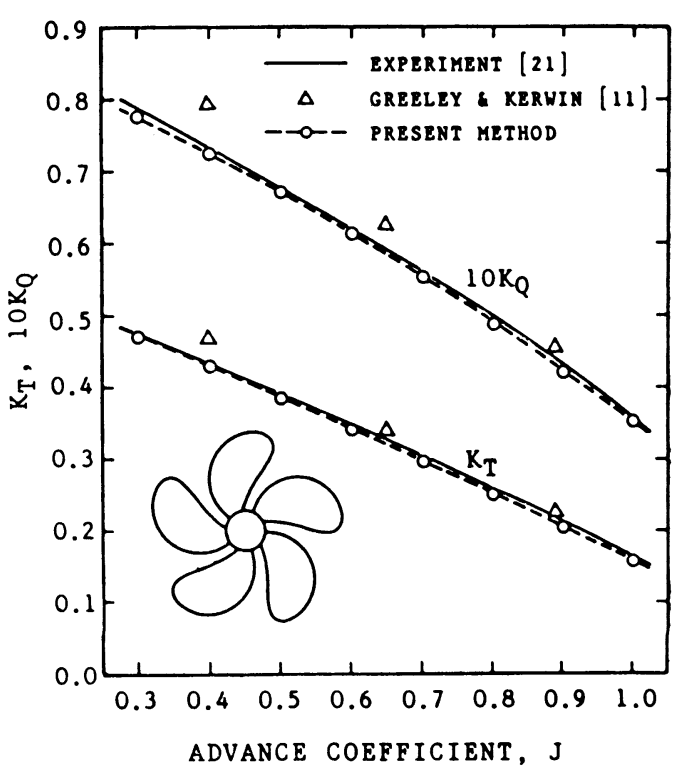

Fig. 10 Comparison of measured and calculated open-water characteristics of DTNSRDC Propeller 4382 (36-deg skew)

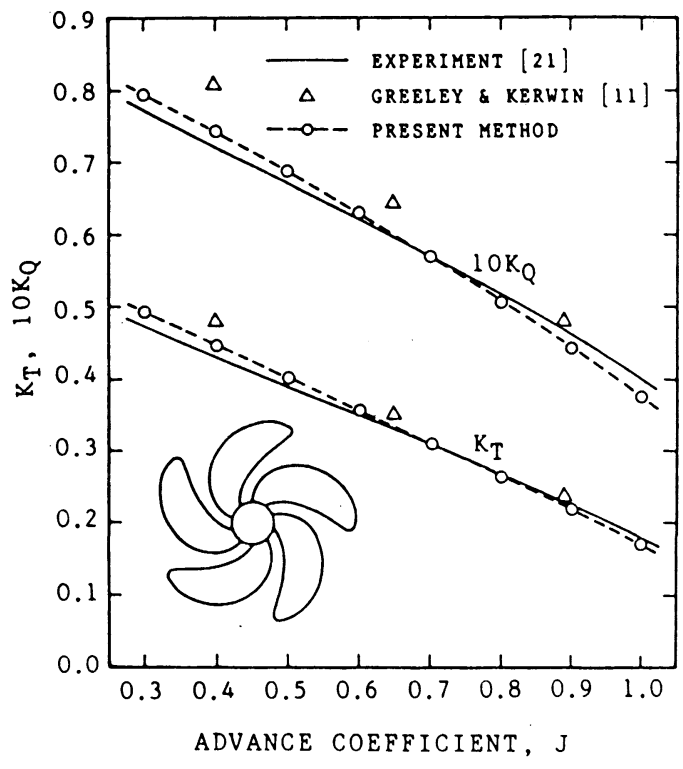

Fig. 11 Comparison of measured and calculated open-water characteristics of DTNSRDC Propeller 4383 (72-deg skew)

\subsection{Pressure Distribution on Propeller Blade}

Comparisons of calculated pressure distribution were made with some of available measured data for the following propellers, i.e. Propeller 


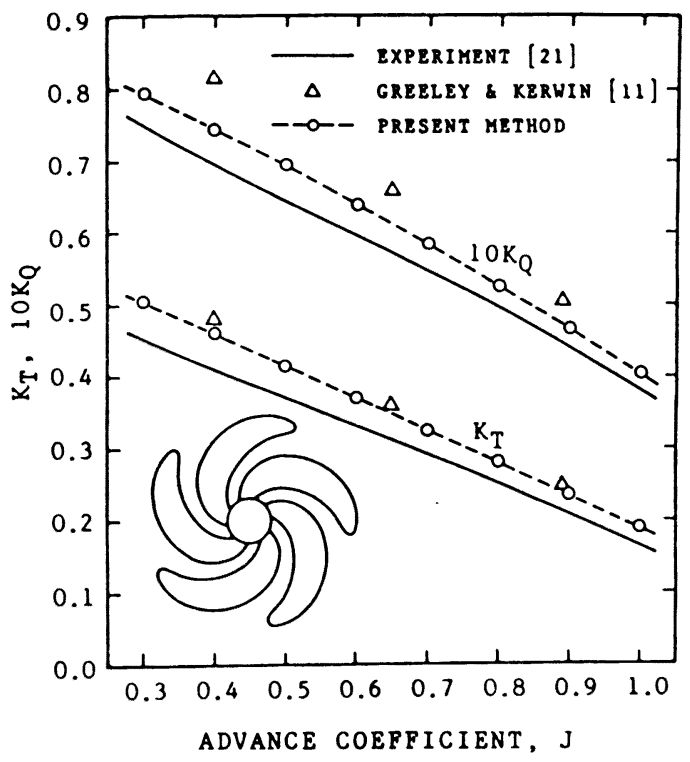

Fig. 12 Comparison of measured and calculated open-water characteristics of DTNSRDC Propeller 4384 (108-deg skew)

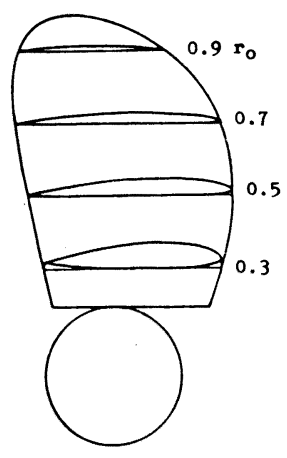

$\begin{array}{lc}\text { DIAMETER (mm) } & 268.0 \\ \text { PITCH RATIO } & 1.0000 \\ \text { EXPANDED AREA RATIO } & 0.6520 \\ \text { BOSS RATIO } & 0.1910 \\ \text { NUMBER OP BLADES } & 5 \\ \text { RAKE ANGLE (DEG.) } & 8 \\ & \\ \end{array}$

Fig. 13 Principal particulars and blade geometry of Propeller $\mathrm{CP}(\mathrm{A})$

$\mathrm{CP}(\mathrm{A})^{22)}$ and the NSMB Propeller ${ }^{23)}$.

$\mathrm{CP}(\mathrm{A})$ is a conventional propeller designed for a container ship. Expanded blade contour and blade sections are shown in Fig. 13 together with its principal particulars. Measurement of pressure distribution on the blade surface was carried out in the Nagasaki Experimental Tank, MHI, with the pressure transducers PD107-1F (TOYODA KOKI) mounted on the propeller blade. The diaphragm of the pressure transducers is 0.5 $\mathrm{mm} \times 1.0 \mathrm{~mm}$.

Measured and calculated pressure distribution at $r / r_{0}=0.75$ and 0.88 at the advance coefficient $J=0.7$ are compared in Fig. 14 . The calculated

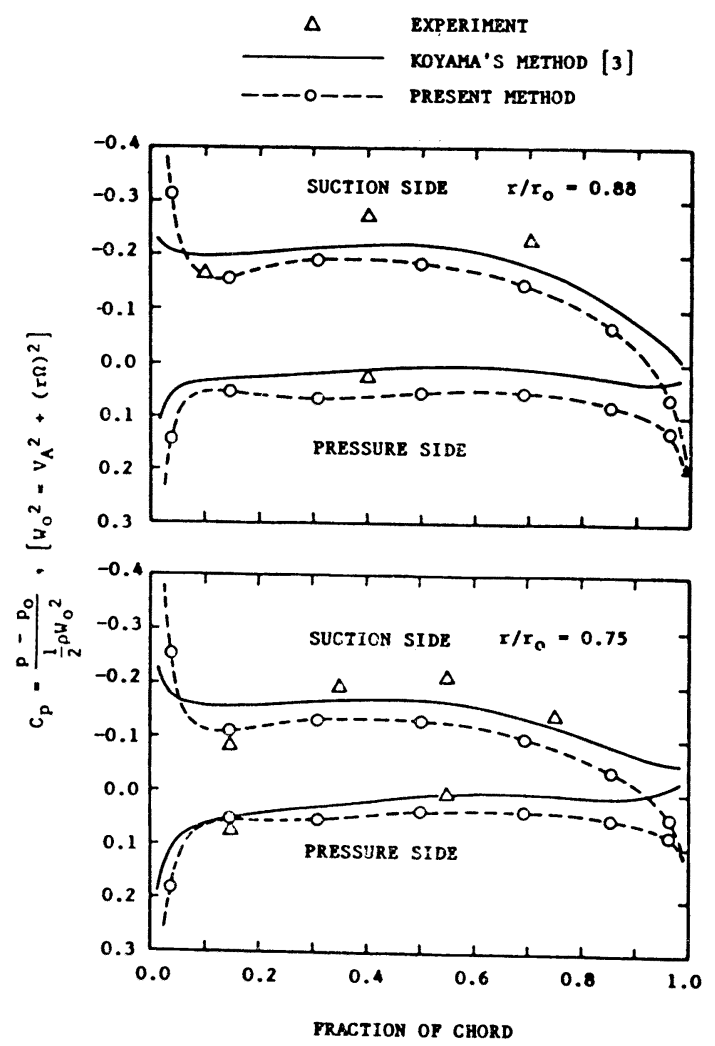

Fig. 14 Comparison of measured and calculated pressure distribution on Propeller $\mathrm{CP}(\mathrm{A})$ at $\mathrm{J}=0.7$

results by Koyama's $\mathrm{MFM}^{3)}$ with equivalent twodimensional wing method ${ }^{2)}$ are also shown in Fig. 14. The calculated pressure distributions by the present method are higher than the measured data on both sides of blade, while calculations by Koyama's method are a little lower than those of present method and are closer to the measured data. Blade loadings (pressure differences between both sides of blade) are nearly equal for the both methods. In the method of equivalent two-dimensional wing the thickness of blade section is treated exactly by Moriya's conformal mapping method ${ }^{24)}$. In the present method, approximate source distribution representing blade thickness is used. This difference in the treatment of blade thickness seems to result in the difference in the calculated results by these two methods.

Fig. 15 shows calculated pressure distribution of the NSMB Propeller at $r / r_{0}=0.8$ at advance coefficients $J=0.6$ and 0.4 in comparison with the data measured at NSMB. Calculated results by Kim and Kobayashi's VLM ${ }^{25}$ are also shown in Fig. 15. Calculation by the present method 


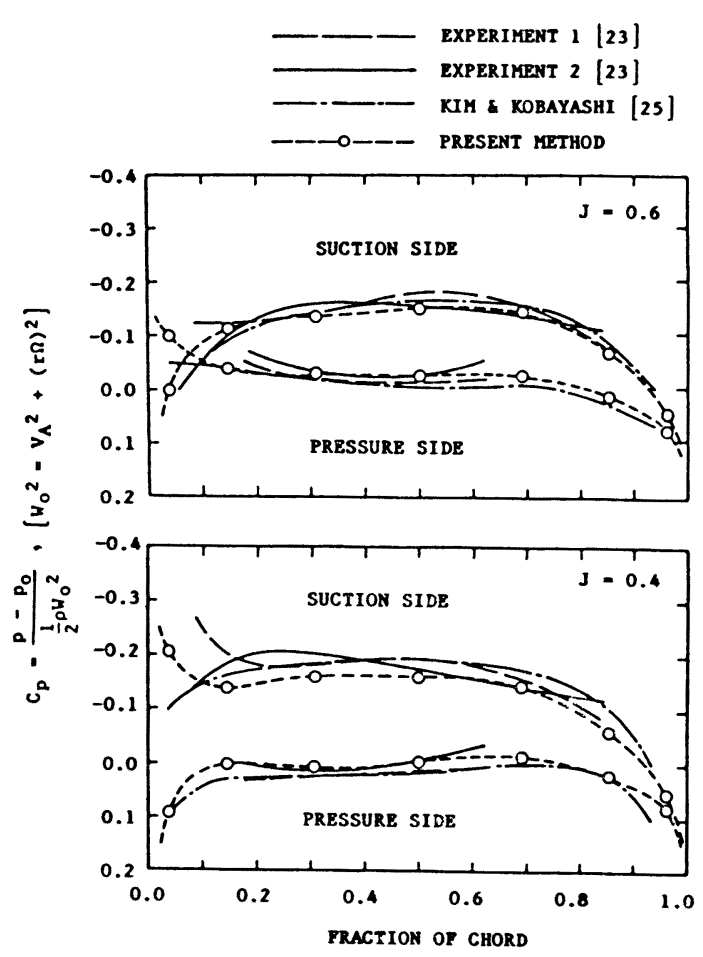

Fig. 15 Comparison of measured and calculated pressure distributions on NSMB Propeller at $r / r_{0}=0.8$

shows good agreement with the measured data and those calculated by Kim and Kobayashi.

\section{Conclusion}

A numerical method to estimate propeller openwater characteristics based on Quasi-Continuous Method (QCM) was presented. Comparisons of propeller open-water characteristics calculated by the present method with measured data showed good agreement for a wide variety of propellers. This indicates that QCM is promising for the improvement of numerical methods in propeller lifting surface theory. However, for the propellers with extremely large skew, the estimation accuracy was not so good.

The present method is still in the early stage of development and following improvements are necessary.

(1) The wake model adopted here was determined based on the preliminary calculations and its geometry is different from that of propeller slip stream actually observed. A more realistic wake model should be formulated based on the measured geometry of slipstream such as contraction, variation of pitch etc.

(2) Viscous effects such as reduction in effective angle of attack and drag coefficient are to be examined theoretically.

(3) Forces acting on source distribution and leading edge suction force should be properly accounted for.

(4) More exact treatment of blade thickness is necessary for the improvement in the calculation accuracy of pressure distribution on blade surface.

\section{Acknowledgement}

The author wishes to express his sincere gratitude to Professor R. Yamazaki of Kyushu University for his continuous encouragement and guidance. Thanks are also due to Mr. K. Tamura, Deputy General Manager of Nagasaki Technical Institute, MHI, for his guidance. Thanks are extended to all the members of the Nagasaki Experimental Tank for their cooperation.

\section{References}

1) Yamazaki, R.: On the Theory of Screw Propellers, Memoirs of the Faculty of Engineering, Kyushu University, Vol. 23, No. 2, 1963.

2) Sugai, K.: A Method for Calculating the Hydrodynamic Characteristics of Marine Propellers, Journal of the Society of Naval Architects of Japan, Vol. 128, Dec. 1970.

3) Koyama, K.: A Numerical Analysis for the Lifting Surface Theory of a Marine Propeller, Journal of the Society of Naval Architects of Japan, Vol. 132, Dec. 1972.

4) Cummings, D. E.: Numerical Prediction of Propeller Characteristics, Journal of Ship Research, Vol. 17, No. 1, March 1973.

5) van Gent, W.: On the Use of Lifting Surface Theory for Moderately and Heavily Loaded Ship Propellers, NSMB Publication No. 536, 1977.

6) Koyama, K.: A Method for Treating the Singularity at the Blade Tip of Marine Propellers, Journal of the Society of Naval Architects of Japan, Vol. 145, June 1979.

7) Tsakonas, S., Jacobs, W. R. and Ali, M. R.: Propeller Blade Pressure Distribution Due to Loading and Thickness Effects, Journal of Ship Research, Vol. 23, No. 2, June 1979.

8) Yamasaki, S.: A Numerical Method for Non-Linear Steady Propeller Lifting Surface and Its Application for Highly Skewed Propeller Design, Transaction of the WestJapan Society of Naval Architects, No. 62, Aug. 1981.

9) Kerwin, J. E. and Lee, C. S.: Prediction of Steady and Unsteady Marine Propeller Performance by Numerical Lifting-Surface Theory, Transaction of the Society of 
Naval Architects and Marine Engineers, Vol. 86, 1978.

10) Yuasa, H.: Application of Numerical Lifting-Surface Theory on Steady Performance of Propeller/Duct System, Journal of the Society of Naval Architects of Japan, Vol. 147, June 1980.

11) Greeley, D. S. and Kerwin, J. E.: Numerical Methods for Propeller Design and Analysis in Steady Flow, Transaction of the Society of Naval Architects and Marine Engineers, Vol. 90, 1982.

12) James, R. M.: On the Remarkable Accuracy of the Vortex Lattice Method, Computer Method in Applied Mechanics and Engineering, Vol. 1, No. 1, 1972.

13) Lan, C. E.: A Quasi-Vortex-Lattice Method in Thin Wing Theory, Journal of Aircraft, Vol. 11, No. 9, Sep. 1974.

14) Zandbergen, P. J., Labrujere, T. E. and Wouters, J. G.: A New Approach to the Numerical Solution of the Equation of Subsonic Lifting Surface Theory, N.L.R Report TR G.49, Nov. 1967.

15) Hough, G. R.: Remarks on Vortex-Lattice Method, Journal of Aircraft, Vol. 10, No. 5, May 1973.

16) Jordan, P. F.: Exact Solutions for Lifting Surfaces, AIAA Journal, Vol. 11, No. 8, Aug. 1973.

17) Abbott, I. H. and von Doenhoff, A E.: Theory of Wing Sections, McGRAW-HILL,
1949.

18) Taniguchi, K. and Sasajima, T.: Scale and Roughness Effects of Propeller Section Drag, Journal of the Society of Naval Architects of Japan, Vol. 133, June 1973.

19) Tamura, K. and Sasajima, T.: Some Investigations on Propeller Open-Water Characteristics for Analysis of Self-Propulsion Factors, Mitsubishi Technical Bulletin, No. 119, March 1977.

20) SR 183, The Shipbuilding Research Association of Japan, March 1983.

21) Boswell, R. J.: Design, Cavitation Performance and Open-Water Performance of a Series of Research Skewed Propellers, DTNSRDC Report 3339, March 1971.

22) Chiba, N. and Nakamura, N.: An Experimental Study on a Highly Skewed Propeller, Technical Review of Mitsubishi Heavy Industries, Vol. 18, No. 2, June 1981.

23) Versmissen, G. G. P. and van Gent, W.: Hydrodynamic Pressure Measurements on a Ship Model Propeller, Proceedings of 14th Symposium on Naval Hydrodynamics, 1983.

24) Moriya, T.: An Introduction to Aerodynamics, Baihukan Book Co. 1959.

25) Kim, K. H. and Kobayashi, S.: Pressure Distribution on Propeller Blade Surface Using Numerical Lifting Surface Theory, Proceedings of Propeller '84 Symposium, May 1984. 